\title{
Levantamento da fauna silvestre no centro de reabilitação do batalhão da polícia militar ambiental nos anos de 20 I0, 20 I I e 2013 no município de Candeias do Jamari-RO
}

Survey of wild fauna in rehabilitation centre of environment military police battalion of 2010,20II and 2013 in the municipality of Candeias Jamari-RO

\author{
Sumara Matos Da Silva', Renato Abreu Lima² \\ ' Faculdades São Lucas, Porto Velho, RO, Brasil \\ 2 Universidade Federal do Amazonas, Manaos, AM, Brasil
}

\begin{abstract}
Resumo
Nosso planeta é habitado por muitos milhões de espécies, e é patrimônio para a humanidade nos aspectos ecológico, científico, econômico e cultural. No Brasil temos a maior diversidade do planeta o que o eleva ao posto de principal nação entre os 17 países de maior biodiversidade. O Batalhão de Polícia Militar Ambiental (BPA) tem a missão de planejar, coordenar e executar o policiamento, visando à proteção dos animais e do meio ambiente. E é no Centro de Reabilitação de Animais Silvestres (CRAS) que os animais apreendidos ou recolhidos são entregues para avaliar sua condição física, tratar esses animais e posteriormente soltá-los no meio ambiente. O estudo teve como objetivo realizar um levantamento das espécies de animais silvestres recolhidas no CRAS/BPA, no período de 2010, 2011 e 2013. Foram analisados registros obtidos a partir de relatórios redigidos pelo CRAS, e foi possível constatar que das 185 espécies, as aves foram às espécies mais predominantes, já que apresentaram, aproximadamente, $52,43 \%$ do número total, seguido dos mamíferos, com aproximadamente, 29,18 $\%$, e por fim, os répteis, com $18,37 \%$.
\end{abstract}

Palavras-chave: animais silvestres, biodiversidade, centro de reabilitação, Rondônia.

\begin{abstract}
Our planet is inhabited by many millions of species, and it is worth to mankind in ecological aspects, scientific, economic and cultural. In Brazil we have the greatest diversity of the planet that rises to the rank of major nation among 17 countries with the greatest biodiversity. The Environmental Military Police Battalion (BPA) mission is to plan, coordinate and implement policing, aiming to protect animals and the environment. And it is at the Center for Rehabilitation of Wild Animals (CRAS) that animals seized or collected are given to assess your physical condition, treat these animals and then release them into the environment. The study aimed to survey the wildlife species collected in CRAS/BPA, from 2010, 2011 and 2013. We analyzed records obtained from reports prepared by CRAS, and it was established that 185 species of birds were the most prevalent species, as presented, approximately $52.43 \%$ of the total, followed by mammals, with approximately $29.18 \%$, and finally, reptiles, with $18.37 \%$.
\end{abstract}

Keywords: wildlife, biodiversity, rehabilitation center, Rondônia. 


\section{INTRODUÇÃO}

A fauna é patrimônio para a humanidade nos aspectos ecológico, científico, econômico e cultural, porém, uma parcela ampla da sociedade não reconhece esse valor. A maioria da população provavelmente não possui consciência da importância ambiental das diferentes espécies na estruturação, na manutenção e no equilíbrio biológico dos ecossistemas, essencial para todas as formas de vida (VIDOLIN et al., 2004).

Nosso planeta é habitado por muitos milhões de espécies. Provavelmente nunca saberemos exatamente quantas, pois os esforços dos cientistas em catalogá-las são contrabalanceados pelas altas taxas de extinção impostas pelo homem, e muitas espécies estão sendo perdidas antes mesmo de serem descobertas e nomeadas (FONTANA et al., 2003).

O Brasil abriga a maior biodiversidade do planeta. Esta abundante variedade de vida que se traduz em mais de $20 \%$ do número total de espécies da Terra, eleva o Brasil ao posto de principal nação entre os 17 países megadiversos (MMA, 2013). E conforme o disposto no Art. 29 da Lei de $n^{\circ} 9.605$, de 12 de fevereiro de 1998, conhecida como Lei dos Crimes contra a Fauna, considera-se crime, matar, perseguir, caçar, apanhar, utilizar espécimes da fauna silvestre, nativos ou em rota migratória, sem a devida permissão, licença ou autorização da autoridade competente (BRASIL, 1998).

Assistimos nas últimas décadas uma verdadeira devastação em nome do progresso, as florestas estão diminuindo velozmente e com elas os animais que dela dependem para sobreviver. A atividade humana tem ameaçado habitat, e consequentemente espécies de animais, isso se deve principalmente a abertura de vias de acesso que facilitam práticas desregradas de atividades como a pecuária, a agricultura, a construção de hidrelétricas e a retirada ilegal de madeira da floresta (LAURENCE et al., 1998).

Mesmo os animais silvestres sendo protegidos pela força da lei, estima-se que, a cada ano, 12 milhões de espécimes sejam retirados das florestas brasileiras (RENCTAS, 2001) para abastecer o tráfico interno e externo. Por ser a fiscalização predominantemente reativa, os dados podem ser muito maiores, pois apenas pequena parte desses animais e seus subprodutos são efetivamente apreendidos (BORGES et al., 2006)

Dentre as estratégias conhecidas para a conservação de ambientes naturais, as unidades de conservação são um dos principais instrumentos de política para a preservação da diversidade biológica e a garantia da conservação de amostras representativas dos ecossistemas (LUIZ, 2008).

Os centros de triagem têm por objetivo recepcionar, e triar os animais resgatados ou apreendidos pelos órgãos fiscalizadores e ainda pelos espécimes provenientes de entrega voluntária. Além disso, é responsável pela guarda desses animais, por seu tratamento clínico, por sua manutenção e por sua destinação final, seja essa uma soltura em áreas de preservação, ou destinação para criadores cadastrados como zoológicos e parques (FRANCO et al., 2012).

De acordo com Jones (1990) e Gipps (1991), a reintrodução tem como objetivos: aumentar o tempo de sobrevivência das espécies; restabelecer espécies-chave (ecologicamente ou culturalmente); aumentar o montante da biodiversidade; e buscar o equilíbrio ambiental acima de tudo.

O Policiamento Florestal da PMRO foi criado através do Convênio n ${ }^{\circ}$ 164/PGE/84, firmado entre IBDF - Instituto Brasileiro do Desenvolvimento Florestal e o Governo do Estado de Rondônia, com a interferência da Polícia Militar do Estado de Rondônia, órgão encarregado de sua execução.

O Batalhão de Polícia Militar Ambiental é uma unidade especializada da Polícia Militar de Rondônia. O órgão tem atuação no Estado, com responsabilidade e competência. Sediada no município de Candeias do Jamari há 27 anos tem a missão de planejar, coordenar e executar o policiamento ostensivo terrestre e aquático, agindo preventivamente e repressivamente, de forma integrada, visando à proteção do meio ambiente (recursos hídricos, flora, recursos minerais, faunas aquática e terrestre), buscando a conscientização, o desenvolvimento sustentável e a qualidade de vida (POLÍCIA MILITAR AMBIENTAL, 2013). Portanto, este trabalho teve como objetivo realizar um levantamento das espécies de animais silvestres resgatados no Centro de Reabilitação de Animais Silvestres (CRAS) do Batalhão de Polícia Militar Ambiental (BPA).

\section{METODOLOGIA}

O presente trabalho foi desenvolvido através de uma análise documental proveniente do Cen- 
tro de Reabilitação de Animais Silvestres - CRAS, sendo este um dos setores do Batalhão de Polícia Ambiental - BPA, que está situado no município de Candeias do Jamari no Estado de Rondônia, BR $364, \mathrm{~km} 22$.

Os dados utilizados para este levantamento foram obtidos por meio de relatórios mensais redigidos como quadro de atividades no CRAS a cada ano, e que foram disponibilizados para esta pesquisa. Os relatórios utilizados foram dos anos de 2010, 2011 e 2013. Os dados do ano de 2012 não foram incluídos no presente trabalho devido a problemas ocorridos na análise dos dados do relatório, embora quantificados, os dados não estavam disponíveis e o pesquisador não teve acesso ao relatório.

Nestes relatórios constavam dados sobre o dia de entrada e saída dos animais, nomes populares, ação empregada, condições físicas e o histórico dos animais. A partir desses dados, foram analisadas as quantidades de registros e agrupados em diferentes espécies para a construção de outra tabela com dados coletados sobre nome popular das espécies.

Posteriormente, os dados formam ordenados por grupo animal para a identificação das espécies, na qual encontramos o grupo taxonômico, nome popular, nome científico e o ano(s) no qual a espécie deu entrada.

Os espécimes foram identificados e consultados de acordo com a taxonomia dos grupos zoológicos, seguindo as classificações propostas por Bérnils (2009), CBRO (2007) e Reis et al. (2006).

\section{RESULTADOS E DISCUSSÃO}

Foram contabilizados informações dos anos de 2010, 2011 e 2013 (sendo que este último ano o relatório só informa até o mês de Agosto), para a realização do levantamento das espécies apreendidas, capturadas ou recebidas no CRAS/BPA, durante este período, o que resultou em um total de 185 espécies diferentes.

A análise das espécies mostrou que o grupo das Aves predominou sobre os demais (Tabela 1) apresentando um total de 97 espécies, correspondendo a aproximadamente 52,43\% do número total de espécies. Seguido dos Mamíferos com 54 espécies, que equivale a aproximadamente a 29,18\% do número total de espécies. E por último os Répteis com 34 espécies, o que equivale a aproximadamente $18,37 \%$ do número total de espécies.

Tabela 1. Número de espécies diferentes por classes de animais silvestres encontrados anualmente no estado de Rondônia pelo Batalhão de Polícia Militar Ambiental de Rondônia, no período de 2010, 2011 e 2013.

\begin{tabular}{cccc}
\hline ANO & & \multicolumn{2}{c}{ CLASSE DE ANIMAIS } \\
& RÉPTEIS & AVES & MAMÍFEROS \\
2010 & 8 & 31 & 25 \\
2011 & 15 & 45 & 21 \\
2013 & 11 & 21 & 8 \\
Total & 34 & 97 & 54 \\
\hline
\end{tabular}

O mesmo verifica-se em trabalhos realizados no âmbito nacional onde as aves representam cerca de $82 \%$ do total de apreensões, sendo as da ordem Passeriformes as mais apreendidas (RENCTAS, 2001; IBAMA, 2002; IBGE, 2004). Isto já era esperado, visto que os Passeriformes compreendem a maioria das aves canoras, sendo os mais comuns em cativeiro de todo o mundo, estando mais de dois milhões dessas aves envolvidas no mercado mundial anualmente (RENCTAS, 2001), o que demonstra a preferência dos comerciantes e da população por estas aves.

Ao avaliar os 625 registros de entrada, pode-se verificar que em 2010, obteve-se 207 indivíduos (33,12\%), em 2011, 309 indivíduos (52,64\%) e em 2013, 89 indivíduos (14,24\%).

A grande discrepância entre os dados pode ser decorrente de flutuações, tanto nas quantidades traficadas quanto, principalmente, na intensidade e rigor da fiscalização de estradas, feiras e aeroportos 
que, de forma geral, carece de sistematização, planejamento adequado e recursos suficientes para o êxito das operações, como retratado pelo IBGE (2004).

Dentre as espécies que mais deram entrada no CRAS em 2010, estão: o Jabuti (Chelonoidis sp.), com 16 indivíduos, o Macaco Prego (Sapajus apella), com 14 indivíduos e o Papagaio (Amazona sp.), com 14 indivíduos. E entre as espécies que menos deram entrada estão: a Onça Pintada (Panthera onca), Ave Jacú (Penelope obscura) e Cobra Caninana (Spilotes pullatus), com 01 indivíduo em cada espécie (Tabela 2).

Em 2011 as espécies que mais deram entrada foram: Cobra Jiboia (Boa constrictor), com 34 indivíduos, Pássaro Curió (Sporophila angolensis), com 23 indivíduos e Tracajá (Podocnemis unifilis), com 17 indivíduos. E as espécies que menos deu entrada, foram Tamanduá Mirim (Tamandua tetradactyla), Pássaro Japiim (Cacicus cela) e Jabuti-Piranga (Geochelone carbonaria), com 01 indivíduo em cada espécie.

As frequências registradas por estes autores para o gênero Sporophila foram 23,81\% (ROCHA et al., 2006) e 47\% (COSTA, 2005). Nacionalmente, também é verificado o predomínio deste gênero, com 6.046 espécimes apreendidos nos anos de 1999 e 2000 em todo o Brasil, o que corresponde a $16,53 \%$ do total de aves apreendidas nesse período (RENCTAS, 2001).

Os produtos da fauna silvestre são muito utilizados para fabricar adornos e artesanato. As espécies envolvidas variam ao longo dos tempos, de acordo com os costumes e os mercados da moda. Normalmente, são comercializados couros, peles, penas, garras, presas, além de diversas outras partes de animais. Todos esses produtos entram no mercado de moda e suvenirs para turistas (RENCTAS, 2001).

Enquanto que em 2013 entre os animais que mais deram entrada estão: o Pássaro Curió (Sporophila angolensis), com 20 indivíduos, o Papagaio Verdadeiro (Amazona aestiva), com 08 indivíduos e a Cobra Jiboia (Boa constrictor), com 05 indivíduos. E entre as que menos deram entrada, estão: o Gavião (Chondrohierax uncinatuz), Cágado Barbicha (Mesoclemmys gibba) e Tamanduaí (Cyclopes didactylus), com 01 indivíduo em cada espécie.

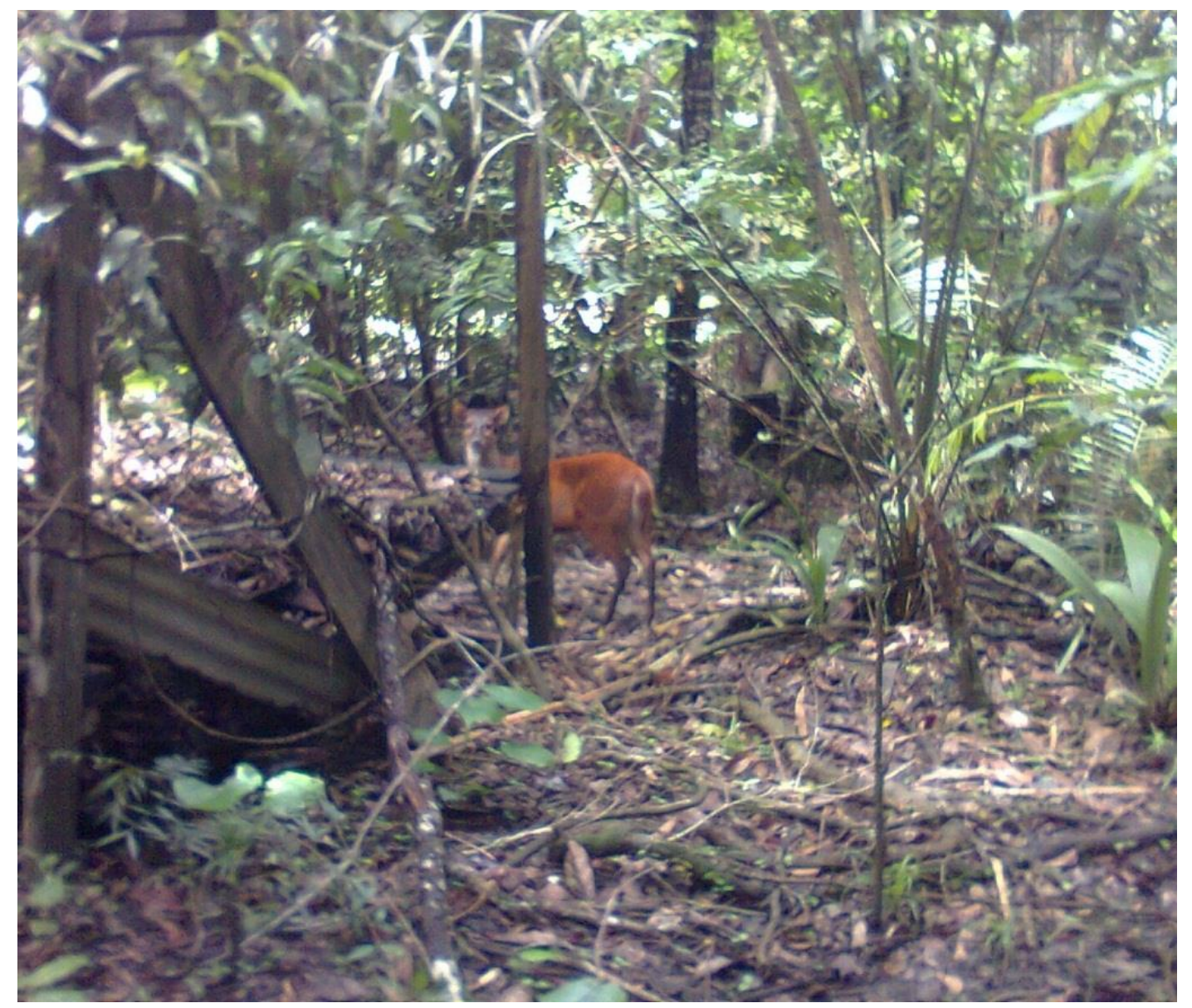

Figura 1. Veado mateiro (Mazama americana, Erxleben, 1777). 


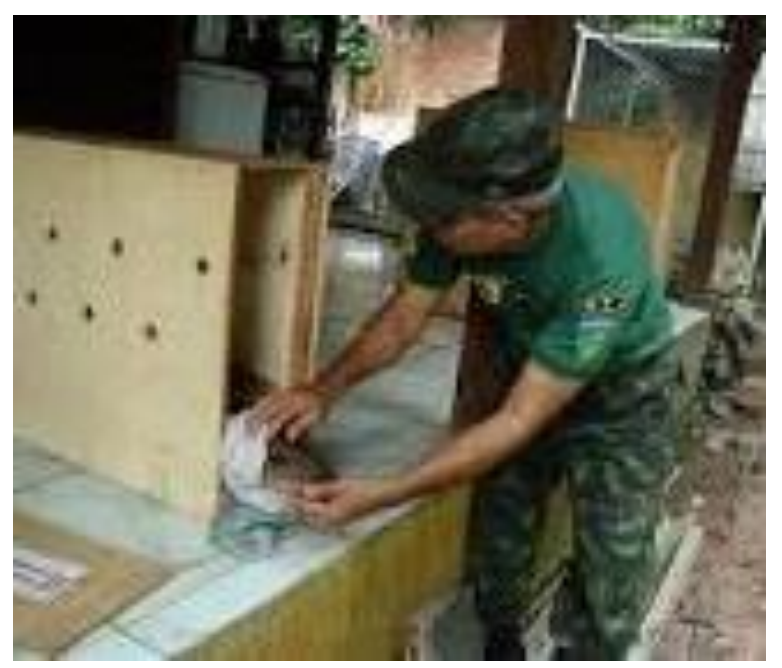

Figura 2. Atividade desenvolvida pelo CRAS: atendimento de animais recebidos no CRAS.

A destinação adequada da fauna silvestre apreendida apresenta-se como um dos principais problemas a serem resolvidos pelos órgãos ambientais (VIDOLIN et al., 2004). Além disso, as atitudes e cuidados governamentais quanto ao uso e à destinação da fauna apreendida devem ser avaliados de forma técnica, legal e ética, sendo obrigação das autoridades constituídas proporcionarem destino apropriado aos animais apreendidos, viabilizando a implantação de locais adequadamente constituídos e equipados, onde sejam efetuadas a identificação e a triagem dos exemplares, além da reabilitação ou da destinação para programas de criação e manutenção em cativeiro (BASTOS et al., 2008).

A maior ameaça à sobrevivência dos animais é a destruição dos hábitats naturais e sua substituição por áreas alteradas pela presença do homem, como plantações, criações de animais domésticos e áreas urbanas (TERBORGH, 1974; GROOMBRIDGE, 1992; PRIMACK \& RODRIGUES, 2001). Esse fator de ameaça atinge magnitude ainda maior quando é observado que a velocidade de destruição do ambiente é maior do que o avanço dos estudos sobre biologia e ecologia in situ ou sobre a reprodução em cativeiro das espécies. Ou seja, corre-se o risco de reduzir tanto os ambientes naturais das espécies que não haverá área disponível para reintrodução das mesmas (PRIMACK; RODRIGUES, 2001). Portanto, é de caráter urgente realizar esforços para a manutenção dos ambientes naturais paralelamente aos estudos das espécies em vida livre e em cativeiro.

Tabela 2. Relação das espécies de animais silvestres encontrados no estado de Rondônia pelo Batalhão de Polícia Militar Ambiental de Rondônia, no período de 2010, 2011 e 2013.

$\begin{array}{clll}\begin{array}{c}\text { Grupo } \\ \text { taxonômico } \\ \text { Mamíferos }\end{array} & \text { Nome popular } & \text { Nome científico } & \text { Ano } \\ & \text { Onça pintada } & \text { Panthera onca } & 2010 \\ & \text { Quati } & \text { (Linnaeus, 1758) } & \\ & & \text { Nasua nasua } & 2010 / 2011 \\ & \text { Macaco prego } & \text { (Linnaeus, 1766) } & \\ & & \text { Sapajus apella } & 2010 / 2011 \\ & & \text { (Kers, 1792) } & \end{array}$


Tabela 2. continuação...

\begin{tabular}{|c|c|c|c|}
\hline \multirow{32}{*}{$\begin{array}{c}\text { Grupo } \\
\text { taxonômico } \\
\text { Mamíferos }\end{array}$} & \multirow{4}{*}{$\begin{array}{l}\text { Nome popular } \\
\text { Veado mateiro }\end{array}$} & \multirow{4}{*}{$\begin{array}{l}\text { Nome científico } \\
\text { Mazama americana } \\
\text { (Erxleben, 1777) }\end{array}$} & \multirow[t]{2}{*}{ Ano } \\
\hline & & & \\
\hline & & & $2010 / 2011$ \\
\hline & & & \\
\hline & \multirow[t]{2}{*}{ Porco caititu } & Pecari tajacu & $2010 / 2011 / 2013$ \\
\hline & & (Linnaeus, 1758) & \\
\hline & \multirow[t]{2}{*}{ Macaco zog zog } & Callicebus dubius & 2010/2011 \\
\hline & & (Hershkovitz, 1988) & \\
\hline & \multirow[t]{2}{*}{ Macaco aranha } & Ateles marginatus & $2010 / 2011$ \\
\hline & & (Geoffroy, 1809) & \\
\hline & \multirow[t]{2}{*}{ Sagui-tufo-preto } & Callithrix penicillata & 2010 \\
\hline & & (Hershkovitz, 1977) & \\
\hline & \multirow[t]{2}{*}{ Bicho preguiça } & Bradypus variegatus & 2010/2011/2013 \\
\hline & & (Schinz, 1825) & \\
\hline & \multirow{2}{*}{$\begin{array}{l}\text { Macaco-prego- } \\
\text { galego }\end{array}$} & Sapajus flavius & 2010 \\
\hline & & (Schreber, 1774) & \\
\hline & \multirow[t]{2}{*}{ Cutia } & Dasyprocta fuliginosa & 2010/2011 \\
\hline & & (Wagler, 1832) & \\
\hline & \multirow[t]{2}{*}{ Jaguatirica } & Leopardus pardalis & $2010 / 2011 / 2013$ \\
\hline & & (Linnaeus, 1758) & \\
\hline & \multirow[t]{2}{*}{ Porco queixada } & Tayassu pecari & 2010 \\
\hline & & (Link, 1795) & \\
\hline & \multirow{2}{*}{$\begin{array}{l}\text { Sagui-de-boca- } \\
\text { branca }\end{array}$} & Saguinus mystax & 2010 \\
\hline & & (Spix, 1823) & \\
\hline & \multirow[t]{2}{*}{ Preá } & Cavia aperea & 2010 \\
\hline & & (Erxleben, 1777) & \\
\hline & \multirow[t]{2}{*}{ Gato mourisco } & Puma yagoauroundi & 2010 \\
\hline & & (Geoffroy, 1803) & \\
\hline & \multirow[t]{2}{*}{ Ouriço cacheiro } & Coendou prehensilis & 2010/2011 \\
\hline & & (Linnaeus, 1758) & \\
\hline & \multirow[t]{2}{*}{ Tatu peba } & Euphractus sexcinctus & 2010 \\
\hline & & (Linnaeus, 1758) & \\
\hline
\end{tabular}


Tabela 2. continuação...

$\begin{array}{lll}\text { Grupo } & \text { Nome popular } & \text { Nome científico } \\ \text { taxonômico } & & \\ \text { Mamíferos } & & \end{array}$

\begin{tabular}{|c|c|c|}
\hline Tamanduá mirim & $\begin{array}{l}\text { Tamandua tetradactyla } \\
\text { (Linnaeus, 1758) }\end{array}$ & $2010 / 2011 / 2013$ \\
\hline Macaco-da-noite & $\begin{array}{l}\text { Aotus nigriceps } \\
\text { (Dollman, 1909) }\end{array}$ & 2010 \\
\hline Sagui SI & $\begin{array}{l}\text { Simia aurita } \\
\text { (E. Geoffroy Saint- } \\
\text { Hilaire, 1912) }\end{array}$ & 2010 \\
\hline Paca & $\begin{array}{l}\text { Cuniculus paca } \\
\text { (Linnaeus, 1766) }\end{array}$ & $2010 / 2013$ \\
\hline Macaco parauacu & $\begin{array}{l}\text { Pithecia irrorata } \\
\text { (Gray, 1842) }\end{array}$ & 2010 \\
\hline Tamanduá bandeira & $\begin{array}{l}\text { Myrmecophaga } \\
\text { tridactyla }\end{array}$ & 2011 \\
\hline Macaco barrigudo & $\begin{array}{l}\text { (Linnaeus, 1758) } \\
\text { Lagothrix lagotricha } \\
\text { (Humboldt, 1812) }\end{array}$ & 2011 \\
\hline Tatu & Dasypus sp. & 2010 \\
\hline Rato do mato & $\begin{array}{l}\text { Kunsia fronto } \\
\text { (Winge, 1887) }\end{array}$ & 2011 \\
\hline Mucura & $\begin{array}{l}\text { Didelphis marsupialis } \\
\text { (Linnaeus, 1758) }\end{array}$ & 2011 \\
\hline Tatu galinha & $\begin{array}{l}\text { Dasypus novemcinctus } \\
\text { (Linnaeus, 1758) }\end{array}$ & $2011 / 2013$ \\
\hline Raposa cinzenta & $\begin{array}{l}\text { Urocyon } \\
\text { cinereoargenteus }\end{array}$ & 2011 \\
\hline Mico leão cara preta & $\begin{array}{l}\text { (Schreber, 1775) } \\
\text { Leontopithecus caissara } \\
\text { (Lorini \& Persson, } \\
\text { 1990) }\end{array}$ & 2011 \\
\hline Tamanduaí & $\begin{array}{l}\text { Cyclopes didactylus } \\
\text { (Linnaeus, 1758) }\end{array}$ & 2013 \\
\hline
\end{tabular}

continua... 
Tabela 2. continuação...

\begin{tabular}{|c|c|c|c|}
\hline \multirow[t]{15}{*}{$\begin{array}{c}\text { Grupo } \\
\text { taxonômico } \\
\text { Aves }\end{array}$} & Nome popular & Nome científico & Ano \\
\hline & Arara canidé & $\begin{array}{l}\text { Ara ararauna } \\
\text { (Linnaeus, 1758) }\end{array}$ & $2010 / 2011 / 2013$ \\
\hline & Arara canga & $\begin{array}{l}\text { Ara macao } \\
\text { (Linnaeus, 1758) }\end{array}$ & $2010 / 2011 / 2013$ \\
\hline & $\begin{array}{l}\text { Papagaio } \\
\text { Maritaca }\end{array}$ & $\begin{array}{l}\text { Amazona sp. } \\
\text { Aratinga leucophthalmus } \\
\text { (Statius Muller, 1776) }\end{array}$ & $\begin{array}{l}2010 / 2011 / 2013 \\
2010\end{array}$ \\
\hline & Jacú & $\begin{array}{l}\text { Penelope obscura } \\
\text { (Hellmayr, 1914) }\end{array}$ & 2010 \\
\hline & Juriti & $\begin{array}{l}\text { Leptotila verreauxi } \\
\text { (Bonaparte, 1855) }\end{array}$ & $2010 / 2011$ \\
\hline & Falcão cauré & $\begin{array}{l}\text { Falco rufigularis } \\
\text { (Daudin, 1800) }\end{array}$ & 2010 \\
\hline & Pássaro curió & $\begin{array}{l}\text { Sporophila angolensis } \\
\text { (Linnaeus, 1766) }\end{array}$ & 2010/2011/2013 \\
\hline & Gavião carijó & $\begin{array}{l}\text { Rupornis magnirostris } \\
\text { (Gmelin, 1788) }\end{array}$ & $2010 / 2011$ \\
\hline & Rolinha roxa & $\begin{array}{l}\text { Columbina talpacoti } \\
\text { (Temminck, 1811) }\end{array}$ & $2010 / 2011$ \\
\hline & Maracanã verde & $\begin{array}{l}\text { Aratinga leucophthalma } \\
\text { (Statius Muller, 1776) }\end{array}$ & $2010 / 2011 / 2013$ \\
\hline & Coruja & $\begin{array}{l}\text { Tyto alba } \\
\text { (Scopoli, 1769) }\end{array}$ & 2010 \\
\hline & Bacurau & $\begin{array}{l}\text { Hydropsalis albicollis } \\
\text { (Gmelin, 1789) }\end{array}$ & $2010 / 2011$ \\
\hline & Coruja buraqueira & $\begin{array}{l}\text { Athene cunicularia } \\
\text { (Molina, 1782) }\end{array}$ & $2010 / 2011$ \\
\hline & $\begin{array}{l}\text { Papagaio de peito } \\
\text { roxo }\end{array}$ & $\begin{array}{l}\text { Amazona vinacea } \\
\text { (Kuhl, 1820) }\end{array}$ & $2010 / 2011$ \\
\hline
\end{tabular}


Tabela 2. continuação...

\begin{tabular}{|c|c|c|c|}
\hline $\begin{array}{c}\text { Grupo } \\
\text { taxonômico } \\
\text { Aves }\end{array}$ & Nome popular & Nome científico & Ano \\
\hline & $\begin{array}{l}\text { Cigarrinha-do- } \\
\text { campo }\end{array}$ & $\begin{array}{l}\text { Ammodramus aurifrons } \\
\text { (Spix, 1825) }\end{array}$ & 2010 \\
\hline & Coruja orelhuda & $\begin{array}{l}\text { Asio clamator } \\
\text { (Vieillot, 1808) }\end{array}$ & 2010 \\
\hline & Papa-capim & $\begin{array}{l}\text { Sporophila schistacea } \\
\text { (Lawrence, 1862) }\end{array}$ & 2010 \\
\hline & $\begin{array}{l}\text { Maracanã-de-cara- } \\
\text { amarela }\end{array}$ & $\begin{array}{l}\text { Orthopsittaca manilata } \\
\text { (Boddaert, 1783) }\end{array}$ & $2010 / 2011 / 2013$ \\
\hline & Açari/tucano & $\begin{array}{l}\text { Ramphastos toco } \\
\text { (Müller, 1776) }\end{array}$ & $2010 / 2011 / 2013$ \\
\hline & Pássaro coleira & $\begin{array}{l}\text { Sporophila caerulescens } \\
\text { (Vieillot, 1823) }\end{array}$ & $2010 / 2011$ \\
\hline & Frango d'água azul & $\begin{array}{l}\text { Porphyrio Martinica } \\
\text { (Linnaeus, 1766) }\end{array}$ & $2010 / 2011$ \\
\hline & Sanhaço & $\begin{array}{l}\text { Thraupis sayaca } \\
\text { (Linnaeus, 1766) }\end{array}$ & 2010 \\
\hline & Gavião tesoura & $\begin{array}{l}\text { Elanoides forficatus } \\
\text { (Linnaeus, 1758) }\end{array}$ & 2010 \\
\hline & Gaviãozinho & $\begin{array}{l}\text { Gampsonyx swainsonii } \\
\text { (Vigors, 1825) }\end{array}$ & $2010 / 2011$ \\
\hline & Bem-te-vi & $\begin{array}{l}\text { Pitangus sulphuratus } \\
\text { (Linnaeus, 1766) }\end{array}$ & $2010 / 2011$ \\
\hline & Coruja suindara & $\begin{array}{l}\text { Tytonidae alba } \\
\text { (Scopoli, 1769) }\end{array}$ & $2010 / 2011$ \\
\hline & Maitaca cab. Azul & $\begin{array}{l}\text { Pionus menstruus } \\
\text { (Linnaeus, 1766) }\end{array}$ & 2010/2011 \\
\hline & Papagaio campeiro & $\begin{array}{l}\text { Amazona ochrocephala } \\
\text { (Gmelin, 1788) }\end{array}$ & 2010 \\
\hline
\end{tabular}


Tabela 2. continuação...

\begin{tabular}{|c|c|c|c|}
\hline $\begin{array}{c}\text { Grupo } \\
\text { taxonômico } \\
\text { Aves }\end{array}$ & Nome popular & Nome científico & Ano \\
\hline & Papagaio moleiro & $\begin{array}{l}\text { Amazona farinosa } \\
\text { (boddaert, 1783) }\end{array}$ & $2010 / 2011 / 2013$ \\
\hline & Garça branca & $\begin{array}{l}\text { Ardea alba } \\
\text { (Linnaeus, 1758) }\end{array}$ & 2011 \\
\hline & Japiim & $\begin{array}{l}\text { Cacicus cela } \\
\text { (Linnaeus, 1758) }\end{array}$ & 2011 \\
\hline & Periquito verde & $\begin{array}{l}\text { Hidromedusa tectfera } \\
\text { (Rafinesque, 1815) }\end{array}$ & 2011 \\
\hline & Periquito estrela & $\begin{array}{l}\text { Aratinga aurea } \\
\text { (Gmelin, 1788) }\end{array}$ & $2011 / 2013$ \\
\hline & Coleira parda & $\begin{array}{l}\text { Sporophila caerulencens } \\
\text { (Vieillot, 1823). }\end{array}$ & 2011 \\
\hline & Peito roxo pardo & $\begin{array}{l}\text { Cotinga cotinga } \\
\text { (Linnaeus, 1766) }\end{array}$ & 2011 \\
\hline & Maracanã bico preto & $\begin{array}{l}\text { Ramphastos vitellinus } \\
\text { (Lichtenstein, 1823) }\end{array}$ & 2011 \\
\hline & Maracanã & $\begin{array}{l}\text { Primolius maracanã } \\
\text { (Vieillot, 1816) }\end{array}$ & $2011 / 2013$ \\
\hline & Papagaio verdadeiro & $\begin{array}{l}\text { Amazona aestiva } \\
\text { (Linnaeus, 1758) }\end{array}$ & $2011 / 2013$ \\
\hline & Iraúna grande & $\begin{array}{l}\text { Scaphidura oryzivora } \\
\text { (Gmelin, 1788) }\end{array}$ & 2011 \\
\hline & Sagui & $\begin{array}{l}\text { Saguinus bicolor } \\
\text { (Spix, 1823) }\end{array}$ & 2011 \\
\hline & Periquito & Aratinga sp. & $2010 / 2011 / 2013$ \\
\hline & Urutau & $\begin{array}{l}\text { Nyctibius griséus } \\
\text { (Gmelin, 1789) }\end{array}$ & 2011 \\
\hline & Gavião & $\begin{array}{l}\text { Chondrohierax } \\
\text { uncinatus } \\
\text { (Temminck, 1822) }\end{array}$ & $2011 / 2013$ \\
\hline
\end{tabular}


Tabela 2. continuação...

\begin{tabular}{|c|c|c|c|}
\hline \multirow[t]{15}{*}{$\begin{array}{c}\text { Grupo } \\
\text { taxonômico } \\
\text { Aves }\end{array}$} & Nome popular & Nome científico & Ano \\
\hline & Urubu & $\begin{array}{l}\text { Coragyps atratus } \\
\text { (Bechstein, 1793) }\end{array}$ & 2011 \\
\hline & Curica & $\begin{array}{l}\text { Amazona amazonica } \\
\text { (Linnaeus, 1766) }\end{array}$ & 2011 \\
\hline & Falcão peregrino & $\begin{array}{l}\text { Falco peregrinus } \\
\text { (Tunstall, 1771) }\end{array}$ & 2011 \\
\hline & Beija flor & $\begin{array}{l}\text { Eupetomena macroura } \\
\text { (Gmelin, 1788) }\end{array}$ & $2011 / 2013$ \\
\hline & Picaparra & $\begin{array}{l}\text { Heliornis fulica } \\
\text { (Boddaert, 1783) }\end{array}$ & 2011 \\
\hline & Arirambinha & $\begin{array}{l}\text { Chloroceryle aenea } \\
\text { (Pallas, 1764) }\end{array}$ & 2011 \\
\hline & Biguá-pato-d' água & $\begin{array}{l}\text { Phalacrocorax } \\
\text { brasilianus } \\
\text { (Gmelin, 1789) }\end{array}$ & 2011 \\
\hline & Pássaro pardal & $\begin{array}{l}\text { Passer domesticus } \\
\text { (Linnaeus, 1758) }\end{array}$ & 2011 \\
\hline & Arara Vermelha & $\begin{array}{l}\text { Ara chloroptecus } \\
\text { (Gray, 1859) }\end{array}$ & 2011 \\
\hline & Arapapá & $\begin{array}{l}\text { C. cochlearius } \\
\text { (Brisson, 1760) }\end{array}$ & 2011 \\
\hline & Pato marreco & $\begin{array}{l}\text { Anas platyrhynchos } \\
\text { (Linnaeus, 1758) }\end{array}$ & 2013 \\
\hline & Anú & $\begin{array}{l}\text { Crotophaga ani } \\
\text { (Linnaeus, 1758) }\end{array}$ & 2013 \\
\hline & Corujão & $\begin{array}{l}\text { Bubo virginianus } \\
\text { (Gmelin, 1788) }\end{array}$ & 2013 \\
\hline & Corujinha & $\begin{array}{l}\text { Megascops atricapilla } \\
\text { (Temminck, 1822) }\end{array}$ & 2013 \\
\hline
\end{tabular}


Tabela 2. continuação...

\begin{tabular}{|c|c|c|c|}
\hline \multirow[t]{5}{*}{$\begin{array}{c}\text { Grupo } \\
\text { taxonômico } \\
\text { Aves }\end{array}$} & Nome popular & Nome científico & Ano \\
\hline & Gavião real & Harpia harpyja & 2013 \\
\hline & & (Linnaeus, 1758) & \\
\hline & Canário da terra & Sicalis flaveola & 2013 \\
\hline & & (Linnaeus, 1766) & \\
\hline \multirow[t]{23}{*}{ Répteis } & Jabuti & Chelonoidis sp. & $2010 / 2011$ \\
\hline & Tracajá & Podocnemis unifilis & $2010 / 2011 / 2013$ \\
\hline & & (Troschel, 1848) & \\
\hline & Jacaré & Caiman sp. & $2010 / 2011$ \\
\hline & Cobra jiboia & Boa constrictor & $2010 / 2011 / 2013$ \\
\hline & & (Linnaeus, 1758) & \\
\hline & Cobra caninana & Spilotes pullatus & $2010 / 2011 / 2013$ \\
\hline & & (Linnaeus, 1758) & \\
\hline & Iguana & Iguana iguana & $2010 / 2011 / 2013$ \\
\hline & & (Linnaeus, 1758) & \\
\hline & Falsa-coral & Oxyrhopus guibei & $2010 / 2011 / 2013$ \\
\hline & & $\begin{array}{l}\text { (Hoge \& Romano, } \\
\text { 1977) }\end{array}$ & \\
\hline & Cobra salamanta & $\begin{array}{l}\text { Epicrates cenchria } \\
\text { crassus }\end{array}$ & 2010 \\
\hline & & (Amaral, 1929) & \\
\hline & Jacaré tinga & Caiman crocodilos & 2011 \\
\hline & & (Linnaeus, 1758) & \\
\hline & Cobra sucuri & Eunectes murinus & $2011 / 2013$ \\
\hline & & (Linnaeus, 1758) & \\
\hline & Cobra jararaca & Bothrops moojeni & 2011 \\
\hline & & (Hoge, 1965) & \\
\hline & Jabuti-piranga & Geochelone carbonária & 2011 \\
\hline & & (Spix, 1824) & \\
\hline & $\begin{array}{l}\text { Cágado-pescoço-de- } \\
\text { cobra }\end{array}$ & $\begin{array}{l}\text { Hidromedusa tectifera } \\
\text { (Cope, 1869) }\end{array}$ & 2011 \\
\hline
\end{tabular}


Tabela 2. continuação...

\begin{tabular}{|c|c|c|c|}
\hline \multirow[t]{8}{*}{$\begin{array}{l}\text { Grupo } \\
\text { taxonômico } \\
\text { Répteis }\end{array}$} & Nome popular & Nome científico & Ano \\
\hline & Cobra verde & $\begin{array}{l}\text { Oxybelis fulgidus } \\
\text { (Daudin, 1803). }\end{array}$ & 2011 \\
\hline & Jabuti tinga & $\begin{array}{l}\text { Geochelone denticulata } \\
\text { (Linnaeus, 1766) }\end{array}$ & $2011 / 2013$ \\
\hline & Cobra pico de jaca & $\begin{array}{l}\text { Lachesis muta } \\
\text { (Linnaeus, 1766) }\end{array}$ & 2011 \\
\hline & Cobra Dipsadidae & $\begin{array}{l}\text { Xenodon dorbignyi } \\
\text { (Duméril Bibron \& } \\
\text { Duméril, 1854) }\end{array}$ & 2013 \\
\hline & Cágado barbicha & $\begin{array}{l}\text { Mesoclemmys gibba } \\
\text { ( Schweigger, 1812) }\end{array}$ & 2013 \\
\hline & Cobra D'água & $\begin{array}{l}\text { Pseudoeryx plicatilis } \\
\text { (Linnaeus, 1758) }\end{array}$ & 2013 \\
\hline & Cobra colubridae & $\begin{array}{l}\text { Megascops atricapilla } \\
\text { (Temminck, 1822) }\end{array}$ & 2013 \\
\hline
\end{tabular}

\section{CONCLUSÕES}

Nesse período de avaliação, o número de animais recebidos pelo CRAS foi elevado, contabilizando 625 registros de entrada, distribuídos em 185 espécies de animais, em especial a classe das aves, com 97 espécies de animais, revela essa classe como a mais preferida pela população, seja ela para o tráfico, criação doméstica ou mesmo em acidentes relacionados aos animais.

Mediante a identificação das espécies de animais silvestres é possível verificar que no estado de Rondônia há uma ampla diversidade de animais, e que estes estão sendo continuamente ameaçados pelas ações humanas, das mais diversas formas, entre elas: desmatamento de seu habitat, criação em cativeiro, maus tratos, acidentes de trânsito (atropelamentos) e tráfico de animais.

Iniciativas de conservação governamentais e da sociedade civil e a educação ambiental podem ser usadas para preservar e garantir a sobrevivência desses animais, e ainda gerar e propagar importantes informações que possam reduzir qualquer tipo de ameaças á esses animais.

\section{AGRADECIMENTOS}

Ao setor Centro de Reabilitação de Animais Silvestres (CRAS) do Batalhão de Polícia Militar Ambiental (BPA), do município de Candeias do Jamari-RO, pela disponibilização dos relatórios para a realização desta pesquisa; E a Instituição de Ensino Faculdade São Lucas. 


\section{REFERÊNCIAS}

BASTOS, L. F.; LUZ, V. L. F.; REIS, I. J.; SOUZA, V. L. Apreensão de espécimes da fauna silvestre em Goiás - situação e destinação. Revista de Biologia Neotropical v. 5, n. 2, p. 51-63, 2008.

BORGES, R. C.; OLIVEIRA, A.; BERNARDO, N.; COSTA, R. M. M. C. Diagnóstico da fauna silvestre apreendida e recolhida pela Polícia Militar de Meio Ambiente de Juiz de Fora, MG (1998 e 1999). Revista Brasileira de Zoociências, v. 8, p.23-33, 2006.

BÉRNILS, R. S. (Org.). Brazilian reptiles - list of species. Curitiba, Sociedade Brasileira de Herpetologia. Disponível em: <http://www.sbherpetologia.org.br/checklist/repteis.htm>. 2009.

BRASIL. Ministério do Meio Ambiente. Lei n 9.605, de 12 de fevereiro de 1998. Lei de Crimes Ambientais. Dispõe sobre as sanções penas e administrativas derivadas de condutas e atividades lesivas ao meio ambiente, e dá outras providências. Brasília, 1998. Disponível em: <http://www.mma.gov.br/port/gab/asin/lei.html >. Acesso em: 17 de outubro de 2013.

COMITÊ BRASILEIRO DE REGISTROS ORNITOLÓGICOS. Listas das aves do Brasil. 10a. Ed. 2011. Disponível em: $<$ http://www.cbro.org.br/CBRO/pdf/AvesBrasil2011.pdf $>$. Acesso em 11 de outubro de 2013.

COSTA, L. P.; LEITE, Y. L.; MENDES, S. L.; DITCHFIELD, A. D. Conservação de mamíferos no Brasil. Megadiversidade, v. 1, n. 1, p.1-10, 2005.

FONTANA, C. S.; BENCKE, G. A; REIS, R. E. Livro vermelho da fauna ameaçada de extinção no Rio Grande do Sul. Porto Alegre: EDIPUCRS, 2003.

FRANCO, M. R.; CÂMARA, F. M.; ROCHA, D. C. C.; SOUZA, R. M.; OLIVEIRA, N. J. F. Animais silvestre apreendidos no período de 2002 a 2007 na macrorregião de Montes Claros, Minas Gerais. Enciclopédia Biosfera, Centro Científico Conhecer, v. 8, n. 14, p. 1007-1018, 2012.

GIPPS, J. H.W. Beyond Captive Breeding: re-introducing endangered mammals to the wild. Oxford: Clarendon Press. Symposia Zoological Society of London. n. 62, 1991.

GROOMBRIDGE, B. Global Biodiversity: status of the earth's living resources. London: Compiled by the World Conservation Monitoring Centre, Cambridge, U.K. Chapman and Hall, 1992.

IBAMA. 2002. Quantitativo de espécimes recebidos nos núcleos de Fauna e Cetas em 2002. Disponível em:<http://www.ibama.gov.br/fauna/tráfico/downloads/dados_2002.pdf> Acesso em: 15 set. 2006.

IBGE. 2004. Indicadores de desenvolvimento sustentável: dimensão ambiental - biodiversidade. Disponível em: <ftp://geoftp.ibge.gov.br/documentos/recursosnaturais/ids/biodiversidade.pdf> Acesso em: 28 dez 2006.

JONES, S. Captive propagation and reintroduction: a strategy for preserving endangered species? Endangered Species Update, n. 8, v. 1, p. 1-88, 1990.

LAURENCE, W. F.; FERREIRA, L. V.; RANKIM-DE-MERONA, J. M; LAURANCE, S. Rain Forest fragmentation and the dynamics of amazoniam tree communities. Ecology, n. 79, v. 6, p. 2032-2040, 1998.

LUIZ, M. R. Ecologia e conservação de mamíferos de médio e grande porte na Reserva Biológica Estadual do Aguaí. Criciúma: Universidade do Extremo Sul Catarinense. 2008

MINISTÉRIO DO MEIO AMBIENTE. Biodiversidade: biodiversidade brasileira. MMA. Disponível em: <http://www.mma.gov.br/biodiversidade/biodiversidade-brasileira>. Acesso em: 16 de outubro de 2013. 
POLÍCIA MILITAR AMBIENTAL. PMRO - BATALHÃO DE POLÍCIA AMBIENTAL. Disponível em: <http: http://www.pmambientalbrasil.org.br/?conteudo=canal\&canal_id=3> Acesso em 15 out 2013.

PRIMACK, R. B.; RODRIGUES, E. Biologia da conservação. Londrina: Ed. Rodrigues, 2001.

RENCTAS. $1^{\circ}$ Relatório nacional sobre o tráfico de fauna silvestre. Brasília: Rede Nacional Contra o Tráfico Silvestre, 2001.

ROCHA, F.M.; NAPOLI, R. P. D.; MIKICH, S. B. Manejo, reabilitação e soltura de mamíferos selvagens. Arquivo de Ciências Veterinárias e Zoológicas, v.9, n.2, p. 105-109, 2006.

TERBORGH, J. Preservation of the natural diversity: the problem of the extinction prone species. BioScience, v. 6, n. 24, p. $715-722,1974$.

VIDOLIN, G. P. P. R.; MANGINI, M. M. B.; MUCHAILH, M. C. Programa estadual de manejo de fauna silvestre apreendida - Estado do Paraná, Brasil. Caderno de Biodiversidade, v. 4, n. 2, p. 37-49, 2004.

COSTA, L. S. Levantamento de mamíferos silvestres de pequeno e médio porte atropelados na BR 101, entre os munícipios de Joinville e Piçarras, Santa Catarina. Bioscience Journal, v. 27, n. 3, p. 666-672, 2011.

EFE, M. A.; FERREIRA, C. M.; OLMOS, F.; MOHR, L. V.; SILVEIRA, L. F. Diretrizes da Sociedade Brasileira de Ornitologia para a destinação de aves silvestres provenientes do tráfico e cativeiro. Revista Brasileira de Ornitologia, v. 14, n. 1, p. 67-72, 2006.

FELKER, R. M.; DORR, A. C.; ROVEDDER, A. P.; PIAZZA, E. M.; DICK, G. Levantamento parcial da avifauna apreendida pelo Escritório Regional do IBAMA de Santa Maria-RS. Revista Eletrônica em Gestão, Educação e Tecnologia Ambiental, v. 11, n. 11, p. 2506-2510, 2013.

FILHO, N.; GAMA, S. L.; CUNHA, S. S. Análise econômica da criação comercial de animais silvestres. Disponível em: <http://www.bnb.gov.br/content/aplicacao/Publicacoes/REN-Numeros_Publicados/docs/ ren_v31_n2_a3.pdf>. Acesso em: 19 de setembro de 2013.

FILHO, S. L. G.; NOGUEIRA, S. S. C. Criação comercial de animais silvestres: produção e comercialização da carne e de subprodutos na região sudeste do Brasil. Revista Econômica do Nordeste, v. 31, n. 2, p. 188-195, 2000.

GOMES, C. C.; OLIVEIRA, R. L. O tráfico internacional de animais: tratamento normativo e a realidade brasileira. Revista Direito e Liberdade, v. 14, n. 2, p. 29-42, 2012.

HENGEMÜHLE, A.; VARGAS, C. Levantamento de mortes de vertebrados silvestres devido a atropelamento em um trecho da Estrada do Mar (RS-389). Biodiversidade Pampeana, v. 6, n. 2, p. 4-10, 2008.

INSTITUTO AMBIENTAL DO PARANÁ. Bichos do Paraná. Guia da fauna paranaense. Curitiba: IAP. 2009. Disponível em: http://www.redeprofauna.pr.gov.br/arquivos/File/biblioteca/bichosdoparana apresentacao.pdf. Acesso em: 06 de outubro de 2013.

IWANAGA, S. Levantamento de mamíferos diurnos de médio e grande porte no Parque Nacional do Jaú: resultados preliminares. Disponível em: < http://www.fva.org.br/dmdocuments/Li2_Cl_Iwanaga\%202004. pdf>. Acesso em: 10 de outubro de 2013.

LEWINSOHN, T. M.; PRADO, P. I. Quantas espécies há no Brasil? Megadiversidade, v. 1, n. 1, p. 1-7, 2005.

LIMA, S. F.; OBARA, A. T. Levantamento de animais silvestres atropelados na BR-277 ás margens do Parque Nacional do Iguaçu: subsídios ao programa multidisciplinar de proteção á fauna. 2004. Disponível em: <http://www.facimed.edu.br/site/revista/pdfs/ba82e1e6e3f3a2c5f546f7e411f815f6.pdf>. Acesso em: 21 de outubro de 2013. 
MACHADO, B.; ROSSO, A. J. A possibilidade educativa no processo de visitação em unidades de conservação. Disponível em: <http: //www.pucpr.br/eventos/educere/educere2008/anais/pdf/588_887.pdf>. Acesso em: 19 de outubro de 2013.

MARINI, M. A; GARCIA, F. I. Conservação de aves no Brasil. Brasília. Brasília: Megadiversidade, v. 1, n. 1, p. 1-8, 2005.

MARQUES, A. A. B.; FONTANA, C. S.; VÉTEZ, E.; BENCKE, G. A.; SCHNEIDER, M.; REIS, R. E. Lista das espécies ameaçadas de extinção no Rio Grande do Sul. Decreto nº 41.672, de 11 de junho de 2002. Porto Alegre: FZB/MCT-PUCRS/PANGEA, 2002. 19p.

MARQUES, A. C.; LAMAS, C. J. E. Taxonomia zoológica no Brasil: estado da arte, expectativas e sugestões de ações futuras. Museu de Zoologia da Universidade de São Paulo, v. 46, n. 13, p. 139-174, 2006.

NASCIMENTO, J. L.; CAMPOS, I. B. Atlas da fauna brasileira ameaçada de extinção em unidades de conservação federais. Instituto Chico Mendes de Conservação da Biodiversidade. Brasília. 2011. 276p.

NASSARO, A. L. F. O policiamento ambiental e o tráfico de animais silvestres no oeste paulista. Fórum Ambiental de Alta Paulista, v. 8, n. 5, p. 18-35, 2012.

ODALIA-RÍMOLI, A.; ARRUDA, E. J.; RÍMOLI, J.; BUENO, N. R.; COSTA, R. B. Biodiversidade, biotecnologia e conservação genética em desenvolvimento local. Revista Internacional de Desenvolvimento Local, v. 1, n. 1 , p. $21-30,2000$.

PAGANO, I. S. A.; SOUZA, A. E. B. A.; WAGNER, P. G. C.; RAMOS, R. T. C. Aves depositadas no Centro de Triagem de Animais Silvestres do IBAMA na Paraíba: uma amostra do tráfico de aves silvestres no estado. Ornithologia, v. 3, n. 2, p. 133-144, 2009.

PAGLIA, A. P.; FONSECA, G. A. B.; RYLANDS, A. B.; HERRMANN, G.; AGUIAR, L. M. S.; CHIARELLO, A. G.; LEITE, Y. L. R.; COSTA, L. P.; SICILIANO, S.; KIERULFF, M. C. M.; MENDES, S. L.; TAVARES, V. C.; MITTERMEIER, R. A.; PALTON, J. L. Lista anotada dos mamíferos do Brasil. $2^{a}$ Ed. Occasional Paper. Conservation International. 2012. Disponível em: <http://www.academia.edu/1541665/ Annotated_Checklist_of_Brazilian_Mammals_2nd_Edition>. Acesso em: 21 de outubro de 2013.

PEREIRA, G. A.; BRITO, M. T. Diversidade das aves silvestres comercializadas nas feiras livres da Região Metropolitana do Recife. Pernambuco. Atualidades Ornitológicas. 2005.

PETERS, F. B.; ROTH, P. R. O.; PEREIRA, M. S.; PISKE, A. D.; CHRISTOFF, A. U. Aspectos da caça e perseguição aplicada à mastofauna na Área de Proteção Ambiental do Ibirapuitã, Rio Grande do Sul, Brasil. Biodiversidade Pampeana, v. 9, n. 1, p. 16-19, 2011. 